\title{
Mujeres investigadoras en las primeras estructuras de organización en ciencias exactas e ingenierías en México de 1900-2000: Estudio Bibliométrico
}

\author{
María Elena Luna Morales* \\ Evelia Luna Morales*
}

\begin{abstract}
Artículo recibido:
4 de enero de 2018

Artículo aceptado:

19 de septiembre de 2018

Artículo de investigación
\end{abstract}

\section{RESUMEN}

Se analiza la producción científica de México registrada en Web of Science de 1900 a 2000 en las áreas de ciencias exactas e ingenierías para caracterizar la forma en que se incorporan las mujeres investigadoras al desarrollo de estos campos de estudio. El trabajo se apoya en el método bibliométrico cuantitativo a través de la aplicación de indicadores de género, producción e impacto científico y el análisis de redes bibliométricas de coautoría. En México, las ciencias exactas e ingenierías registran sus primeras aportaciones durante la primera mitad del siglo XX. Las mujeres lo hicieron a partir de los años 40 a través de laboratorios Syntex donde publicaron sus contribuciones iniciales. La

* Coordinación General de Servicios Bibliográficos, Cinvestav-IPN, México

meluna@cinvestav.mx eluna@cinvestav.mx

INVESTIGACIÓN BIBLIOTECOLÓGICA, vol. 32, núm. 77, octubre/diciembre, 2018, México, ISSN: 2448-8321 pp. 193-215 
participación de las investigadoras se incrementó entre 1960 y 1970 con el reconocimiento de las mujeres en la educación superior y la investigación, así como la creación de distintas instituciones de investigación en el país, además de los cambios de las mujeres con respecto a la elección de carrera. Finalmente, las contribuciones de este trabajo representan una ventaja para la propia comunidad científica, pero también para las organizaciones orientadas a promover la equidad de género y las instituciones que integran investigadoras en este campo de estudio.

Palabras clave: Ciencias Exactas; Ingenierías; México; Estudio Bibliométrico de Género; Investigación Científica

A Bibliometric study of women researchers in early organizational structures of exact sciences and engineering in Mexico from 1900 to 2000

María Elena Luna-Morales and Evelia Luna-Morales

\section{Abstract}

The scientific production of Mexico registered in Web of Science from 1900-2000 in the areas of Exact Sciences and Engineering is analyzed to describe the way in which female researchers are integrated in the development of these fields of study. The paper employs a quantitative bibliometric method, applying indicators of gender, scientific output and impact, and analysis of bibliometric networks of co-authorship. In Mexico, researchers in exact sciences and engineering published their first papers in the first half of the 20 th century, but female researchers working in Syntex laboratories did not publish until the 1940s. As more women joined faculties in higher education and research centers in the $60 \mathrm{~s}$ and $70 \mathrm{~s}$, publication by female researchers began to increase accordingly. This growth also appears to be associated with changes in career choice by women. While the contributions of this work represent an advantage for the scientific community itself, they also are useful for organizations that promote gender equity and institutions that integrate female researchers in this field of study.

Keywords: Exact Sciences; Engineering; Mexico; Bibliometrics, Gender Study; Scientific Research 


\section{INTRODUCCIÓN}

T a participación de las mujeres en las ciencias sigue siendo un tema de Lebate en todos los ámbitos (Buquet-Corleto, 2013; Pollack, 2013). Esta situación ha llamado la atención de distintos autores y la participación de algunas instituciones públicas y privadas que se han interesado por conocer los problemas a los que se enfrentan las investigadoras, como la formación académica, el progreso en la actividad científica y la ocupación de puestos altos, entre otros. Esto se ha reflejado en estudios como el presentado por The Boston Consulting Group (2013) y los producidos por la Organización de las Naciones Unidas (ONU), a través de su organismo especializado en educación, ciencia y cultura (Unesco, 2010).

Estos estudios se complementan con otros trabajos publicados, en los que se da a conocer el antagonismo y la exclusión hacia las mujeres (Sugimoto, 2013), así como la escasa explotación del potencial del género femenino en la actividad científica y tecnológica y la forma en que esto repercute en el desarrollo económico de los países (Mendieta-Ramírez, 2015).

Asimismo, se pueden mencionar distintas propuestas que giran en torno a la implementación de programas de posgrado más atractivos -que llamen la atención de las mujeres durante la elección de carreras- y la modificación de los planes de estudio desde los niveles educativos más bajos, para ir preparando y motivando a las niñas desde pequeñas (Miroux, 2011; Guevara-Ruiseñor y García-López, 2010).

El tema se complica cuando se analiza la participación de las investigadoras en las ciencias exactas e ingenierías, áreas que por mucho tiempo se han considerado del dominio masculino (Guevara-Ruiseñor y García-López, 2010). En estos campos, las mujeres han prosperado de manera impresionante, pero no lo suficiente como para lograr una mayor presencia. A nivel mundial, en física, las mujeres tienen representación de $10 \%$ del universo y en ingenierías, de 30 \% (Unesco, 2017). Según el Sistema Nacional de Investigadores (SNI) de México, entre 2013 y 2015 las mujeres no fueron mayoría en ninguno de los campos que conforman las ciencias exactas e ingenierías.

Didou y Etienne (2011) y Meza-Montes (2005) señalan, en físico-matemáticas y ciencias de la Tierra, que las mujeres representan $18 \%$ del total, en ingenierías, 19 \% y en biología y química, 40 \%. Esta escasa representación ha orientado a las propias mujeres a realizar estudios más reflexivos que permitan identificar la forma en que funcionan los regímenes de género existentes para identificar los factores que las limitan (Narváez, 2016).

Pollack (2013) infiere que la razón por la que aún hay muy pocas mujeres en la ciencia no tiene que ver con la falta de talento, sino por la desestimación 
que las mismas se tienen por los constantes bloqueos de los que son víctima: escasas contrataciones, sueldos bajos, exiguos reconocimientos, poco liderazgo en proyectos y puestos de trabajo, entre otros aspectos (Rossiter, 1982). A lo anterior se suman factores culturales y psicológicos, medio por el que se caracteriza a la mujer como carente de iniciativa (Pollack, 2013). A estos agentes se unen otros de orden político, económico y legal que, en conjunto, determinan el rol de las mujeres en la ciencia y la investigación, sobre todo, cuando se trata de elegir carrera, donde la familia juega un papel fundamental (Guevara-Ruiseñor y García-López, 2010).

\section{La bibliometría en la evaluación de la ciencia}

Los estudios bibliométricos surgieron desde hace más de un siglo. Sin embargo, fue Pritchard (1969) quien dio a conocer el término bibliometría por primera vez en un trabajo publicado en Journal of Documentation. Los estudios de la ciencia con enfoque bibliométrico se incrementaron a partir de la década de los años 70 del siglo XX. El aumento en el número de publicaciones y el exceso de información dio lugar a nuevos estudios orientados a la identificación de patrones de publicación y comunicación científica.

Los estudios métricos se apoyan en distintas teorías y modelos matemáticos con los que se pueden concretar objetivos relacionados con el comportamiento de la información (Gorbea-Portal, 2013). El método más utilizado es el cuantitativo que, en combinación con la estadística, permite cuantificar los datos a través de la aplicación de indicadores bibliométricos (Velasco et al., 2012).

Los avances computacionales y la necesidad de visualizar los resultados de la producción científica desde una perspectiva distinta han conducido a que la bibliometría se relacione con el análisis de redes sociales como una forma de interpretar los aspectos de relación y cooperación entre los integrantes de un conjunto (Pinto, Moreiro-González y Oliveira de Meira, 2009).

Pajek (http://vlado.fmf.uni-lj.si/pub/networks/pajek/), software gratuito para Windows, se ha convertido en una herramienta muy práctica para el análisis y visualización de redes sociales (Batagelj y Mrvar, 2011). El software se apoya en la teoría de grafos: considera los nodos como individuos y las aristas como vínculos y cuenta con un amplio número de métodos que ayudan a determinar patrones de comportamiento en la colaboración científica (Hernández-García, 2014).

El análisis estructural se basa en las subestructuras que conforman una red. Para comprender estas estructuras es importante identificar los componentes que participan en las relaciones: diadas y triadas cuya conexión puede 
dar lugar a grandes estructuras a través de la cohesión que ejercen. El tamaño de los nodos lo determina quien construye la red, y por este medio puede representar distintas variables, entre otras, la producción que registran los autores, el impacto científico y el número de documentos en colaboración (Oscar-Lluch, 2010).

Es muy amplio el número de trabajos que abordan el tema de la participación de las mujeres en los distintos ámbitos y campos de investigación, incluidas las ciencias exactas e ingenierías (Contreras-Gómez et al., 2015; Cepeda-Zetter, González-Brambila y Pérez-Angón, 2017; Buquet-Corleto, 2013; Pollack, 2013). El tema se aborda desde diferentes enfoques: político, social, educativo, investigación y económico; por hombres, mujeres y un variado número de instituciones y organismos públicos y privados (Valle-Díaz Muñoz y Garay-Sánchez, 2012; The Boston Consulting Group, 2013; Moya-Anegón et al., 2007).

Sin embargo, en México y en el área de ciencias exactas e ingeniería, no hay estudios que aborden el tema del género desde el punto de vista bibliométrico, en complemento con el análisis de redes de coautoría para determinar el aprovechamiento de capital científico (Bourdieu, 2003). Es por ello que, en este estudio, se busca analizar la producción científica de México registrada en Web of Science (WoS) de 1900 a 2000 en las áreas de ciencias exactas e ingenierías, para caracterizar la forma en que se incorporan las investigadoras al desarrollo de estos campos de estudio y la manera en que contribuyen a la formación de las estructuras de organización. Cabe aclarar que se toma este periodo de estudio porque los años 90 están identificados como los años de crecimiento y consolidación de la ciencia en México (Luna-Morales, 2012), y en este caso se cubriría la etapa de mayor crecimiento para ver los incrementos de mujeres en las ciencias exactas e ingenierías.

WoS registró que, entre 1900 y 2000, hubo 68415 autores de ciencias exactas e ingenierías. Entre ellos, 4541 eran mujeres de cualquier parte del mundo y 2957 están adscritas a instituciones de México.

\section{Metodología}

A partir de búsquedas en las bases de datos que conforman la colección principal de WoS, se recuperó la producción científica de México de 1900-2000. Las búsquedas se realizaron por Address para recuperar lo publicado bajo "MEXICO", excluyendo "NUEVO MEXICO OR NM". Los resultados se limitaron utilizando los refinamientos que incluye la base de datos, por este medio se seleccionaron los años y las áreas de estudio (física, química, matemáticas y las distintas ingenierías). 
Los registros se recuperaron de 500 en 500 debido a que WoS establece este límite. Se utilizó Microsoft Excel para conformar un archivo único donde se desagregaron uno a uno algunos campos como dirección de adscripción de los autores (C1), correspondencia de los autores (RP), categorías temáticas (SC) y autores (AU), lo anterior para normalizar y conformar catálogos únicos.

Para generar el catálogo de direcciones de autores se separaron, en un archivo independiente en Excel, los campos de afiliación y correspondencia para desagregar y seccionar cada uno de los apartados que los componen: institución principal, departamentos y unidades, subdependencias, laboratorios, secciones y programas, código postal y entidad federativa y país de origen de los autores. Cada campo se completó y normalizó para originar el índice de direcciones constituido por instituciones, dependencias, estados y países.

Para construir el índice de nombres de autores fue indispensable separarlos uno a uno sin perder la relación con el resto de los campos de la referencia bibliográfica. Esta separación permitió reunir en una sola lista todos los nombres de autores, la cual se organizó en forma alfabética, lo que ayudó a agrupar las variantes de nombres y, con ello, conseguir una normalización más rápida, facilitando la clasificación de los autores por género: mujer $(\mathrm{M})$ y hombre $(\mathrm{H})$.

La clasificación de los autores por género se realizó utilizando distintas fuentes de búsqueda: 1) bases de datos: WoS y Scopus; 2) páginas web de universidades públicas y privadas, centros e institutos de investigación nacionales e internacionales; 3) listas de autores en el SNI; 4) anuarios de instituciones; 5) Google Scholar, y 6) búsquedas libres en internet. Con este método, se identificó el género de 68415 autores de un total de 72 701, lo que hace inferir que 4286 no se identificaron.

Los índices construidos se transfirieron a una base de datos en Access como tablas independientes, como una forma para generar consultas a partir de la relación entre tablas. Para el desarrollo de las redes bibliométricas de coautoría se utilizó Pajek.

Para determinar la participación de las mujeres en las estructuras de investigación en ciencias exactas e ingenierías, se crearon tres matrices que dieron lugar a tres redes de coautoría. La primera cubre de 1900-1970, la segunda de 1971-1975 y la tercera de 1976-1980. En estos casos se aplicó un algoritmo de visualización de datos por separación de componentes. De este modo es más fácil identificar las colaboraciones según el número de autores que conforman la estructura de cada red, así como las relaciones entre los autores que participan y los campos de investigación a la que pertenecen. 
Cabe aclarar que, debido al incremento en el número de autores en años posteriores a 1980, se tomó la decisión de no integrar una red por quinquenio como se había planeado originalmente. Sin embargo, para no dejar duda con respecto al incremento de científicas sobre todo en el último periodo del estudio (1990-2000), se desarrolló la red que abarca de 1996 a 2000, donde se muestra únicamente la distribución de autores por género a través del algoritmo de visualización de optimización de filtro solo, que se encarga de encontrar el estado de equilibrio y mostrar la distribución de nodos sin necesidad de estructurar en redes, lo anterior como una forma de evitar la saturación en la visualización de la red. Es importante mencionar que para contribuir a lo anterior y reducir la cifra de autores, se excluyeron los del área de física de partículas y campos integrados a las colaboraciones Big Science con más de 200 coautores. También se descartó la participación de los autores que registran entre uno y cuatro trabajos.

\section{Presentación y ANÁLISIS DE RESUltados}

\section{Producción científica}

La Figura 1 muestra, por serie anual, los incrementos de la producción. La línea con crecimientos más altos corresponde a la producción global, que incluye todas las áreas y todos los autores sin limitación de género. Las líneas referentes a trabajos-investigadoras y trabajos-investigadoras-México son muy parecidas, excepto las variantes que se muestran al final de los años $70 \mathrm{y}$ segunda mitad de los 80 .

Los crecimientos mostrados en los primeros 70 años son producidos por autores (hombres). Se trata de una contribución mínima, debido al bajo número de investigadores que había en esta época. A comienzos de los años 70 se ven cambios en las tres variables analizadas. Los crecimientos se consolidan principalmente durante las siguientes décadas ( 80 y 90). La producción global muestra un aspecto importante, pues dobla la de las investigadoras en general y la de adscritas a instituciones del país. Aunque hay años en los que las líneas se acercan mucho, en general es muy atípico el crecimiento de esta disciplina.

No obstante, es muy probable que los crecimientos mostrados tengan que ver con los siguientes aspectos: 1) implementación de posgrados de estudio; 2) consolidación de grupos de investigación, y 3 ) incrementos en la colaboración científica nacional e internacional como las Big Science (Luna-Morales y Collazo-Reyes, 2002). 


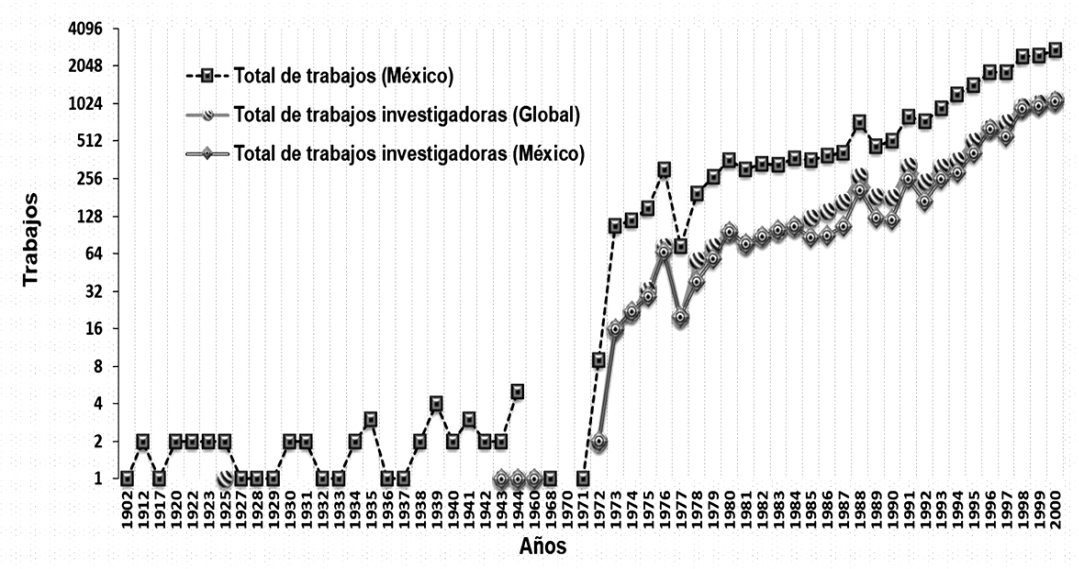

Figura 1. Producción científica anual en ciencias exactas e ingenierías: 1900-2000

La Figura 2 muestra por año la producción científica que los investigadores (mujeres y hombres), integrados a las ciencias exactas e ingenierías, registran en México. Como se mencionó anteriormente, los años 40 marcan los orígenes en el desarrollo de estos campos de estudio, aunque es básicamente en los 70 cuando se observan crecimientos constantes.

De acuerdo con la Figura 2, los investigadores presentan crecimientos mayores a lo largo del periodo de estudio. Las investigadoras hacen lo mismo, pero su producción es dos veces menor que las de los investigadores. Las mujeres alcanzaron los 500 trabajos hasta 1988, rompieron con este tope en 1991 y, en adelante, subieron la producción, pero no lo suficiente para igualar la producción de los investigadores.

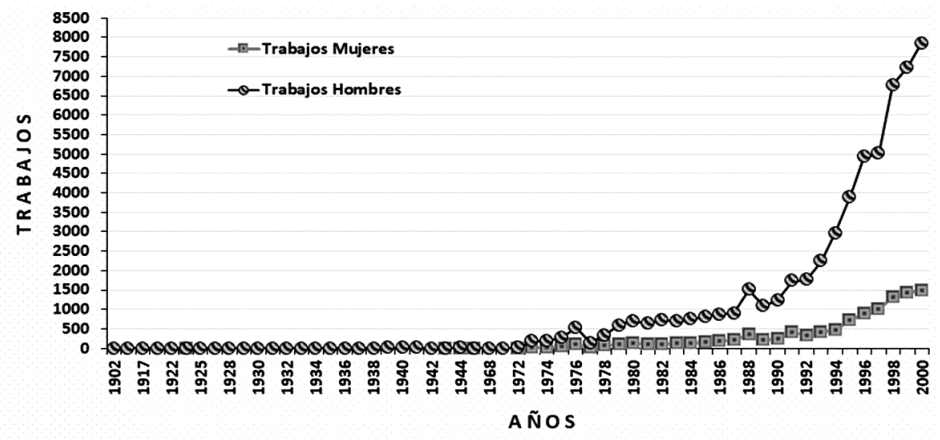

Figura 2. Distribución por serie anual de la producción científica generada por ambos géneros en ciencias exactas e ingenierías: 1900-2000 


\section{Mujeres investigadoras por institución}

La Figura 3 presenta la producción científica generada por investigadoras y por institución, así como la presencia que en años tienen las mujeres. Se distinguen cinco grupos de instituciones que son fundamentales para el progreso que logran las investigadoras en el país:

1) Representado por la UNAM, que muestra la producción más alta generada y tiene 29 años con presencia femenina;

2) Conformado por el Cinvestav y la UAM, ambas instituciones registran crecimientos importantes en producción científica y cuentan con 27 años integrando mujeres en su planta académica y de investigación;

3) Constituido por el Instituto Politécnico Nacional (IPN), el Instituto Nacional de Investigaciones Nucleares (ININ), la Benemérita Universidad Autónoma de Puebla (BUAP) y el Instituto Mexicano del Petróleo (IMP), cuya presencia de mujeres es de 24-27 años y la producción varía entre 250 y 400 trabajos;

4) Compuesto por instituciones que producen de 100 a 200 trabajos, como la Universidad de Sonora (UNISON) y el Instituto Nacional de Astrofísica, Óptica y Electrónica (INAOE), entre otras, y reportan de 13 y 24 años con presencia de mujeres. En este grupo destaca la Universidad de Guanajuato (UGTO) por la producción que registra, pero no por los años que tiene integrando investigadoras, $\mathrm{y}$

5) Formado por las instituciones que registran menos de 100 trabajos y con escasos años con presencia de mujeres, aunque hay excepciones como Syntex, que registra menor producción, pero tienen mayor presencia de mujeres con 57 años. De hecho, es en Syntex donde se identifican las primeras mujeres investigadoras en estas disciplinas.

El resto de las instituciones registran menor producción científica y no rebasan los 20 años con presencia de mujeres, porque son instituciones de reciente creación.

Para un mejor análisis de los datos presentados en la Tabla 1 las instituciones se clasificaron en tres grandes grupos según el tipo de investigación que realizan: 1) tradicionales, por la trayectoria que tienen en la actividad científica y el número de mujeres que integran entre sus afiliados; 2) emergentes, comienzan a incorporar investigadoras a partir de los años 80, y 3 ) intermitentes, afilian investigadoras de manera esporádica. 


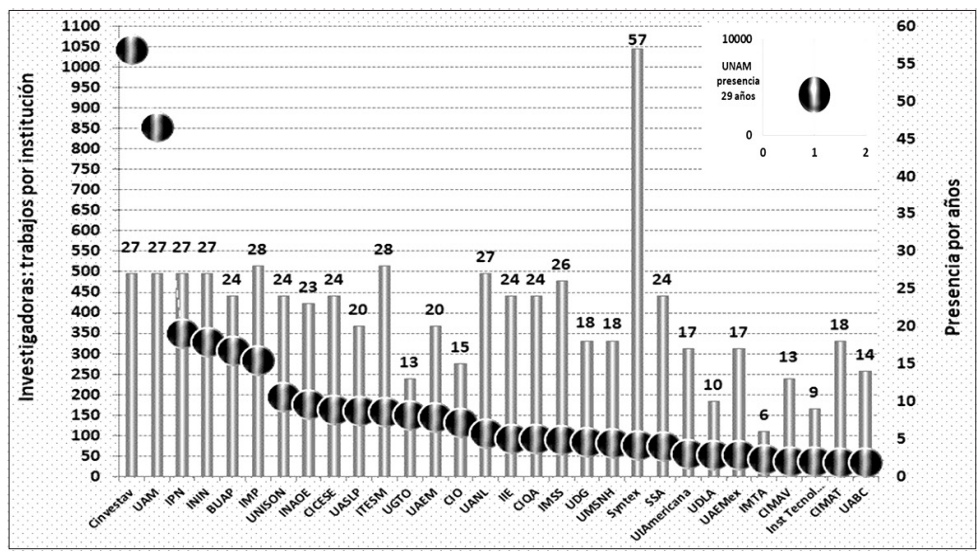

Figura 3. Producción científica por instituciones y presencia de investigadoras en años: 1900-2000

\section{Instituciones tradicionales}

La Tabla 1 muestra por quinquenios y por instituciones la distribución de trabajos publicados por investigadoras, así como la presencia que en años registran las científicas. Cabe aclarar que el valor entre paréntesis corresponde al número de investigadoras y el dato externo a los paréntesis corresponde a los artículos. En este sentido, se pueden identificar las instituciones que más tiempo tienen en la actividad científica y las primeras que integraron mujeres entre sus grupos de investigación.

De 17 instituciones seleccionadas como tradicionales, Syntex y la UNAM son las que tiene más años incorporando investigadoras: 57 y 29 años, respectivamente. No obstante, la UNAM reporta más trabajos publicados por mujeres y mayor número de científicas, mientras que Syntex es de las que menos producción registra y menor número de investigadoras.

Dos casos que también son interesantes son los del Instituto Tecnológico y de Estudios Superiores de Monterrey (ITESM) y el IMP, ya que ambos tienen 28 años incorporando mujeres en sus equipos de investigación. Entre las dos instituciones apenas rebasan los 400 trabajos y reúnen menos de 200 investigadoras en el periodo analizado. Como se observa en la Tabla 1, destaca la presencia de universidades públicas y privadas, centros e institutos de investigación, hospitales e industrias, dando lugar a la clasificación de los siguientes sectores de producción: académica, investigación, salud e industria.

Desde sus orígenes estas instituciones están contribuyendo al crecimiento de la producción nacional y a la fecha continúan siendo base fundamental por la madurez que han desarrollado, con trayectorias de 23 a 27 años trabajando con mujeres investigadoras y con una producción que varía entre 72 y 1042 trabajos. 


\begin{tabular}{|c|c|c|c|c|c|c|c|c|c|}
\hline INSTITUCIÓN & $\begin{array}{c}1900- \\
1970\end{array}$ & $\begin{array}{l}1971- \\
1975\end{array}$ & $\begin{array}{l}1976- \\
1980\end{array}$ & $\begin{array}{l}1981- \\
1985\end{array}$ & $\begin{array}{l}1986- \\
1990\end{array}$ & $\begin{array}{l}1991- \\
1995\end{array}$ & $\begin{array}{l}1996- \\
2000\end{array}$ & TOTALES & $\begin{array}{c}\text { PRESENCIA } \\
\text { X ANNOS }\end{array}$ \\
\hline Syntex & $\begin{array}{c}3 \\
(3)\end{array}$ & $\begin{array}{l}13 \\
(4)\end{array}$ & $\begin{array}{l}17 \\
(7)\end{array}$ & $\begin{array}{l}10 \\
(6)\end{array}$ & $\begin{array}{c}5 \\
(2)\end{array}$ & $\begin{array}{c}16 \\
(13)\end{array}$ & $\begin{array}{l}16 \\
(2)\end{array}$ & 80 & 57 \\
\hline UNAM & & $\begin{array}{c}42 \\
(31)\end{array}$ & $\begin{array}{l}200 \\
(97)\end{array}$ & $\begin{array}{c}307 \\
(144)\end{array}$ & $\begin{array}{c}493 \\
(239)\end{array}$ & $\begin{array}{c}876 \\
(348)\end{array}$ & $\begin{array}{l}2301 \\
(988)\end{array}$ & 4219 & 29 \\
\hline ITESM & & $\begin{array}{c}8 \\
(3)\end{array}$ & $\begin{array}{l}23 \\
(4)\end{array}$ & $\begin{array}{c}8 \\
(5)\end{array}$ & $\begin{array}{c}13 \\
(10)\end{array}$ & $\begin{array}{c}29 \\
(13)\end{array}$ & $\begin{array}{l}75 \\
(42)\end{array}$ & 156 & 28 \\
\hline IMP & & $\begin{array}{c}4 \\
(4)\end{array}$ & $\begin{array}{c}22 \\
(17)\end{array}$ & $\begin{array}{l}18 \\
(9)\end{array}$ & $\begin{array}{c}20 \\
(17)\end{array}$ & $\begin{array}{l}54 \\
(34)\end{array}$ & $\begin{array}{l}166 \\
(96)\end{array}$ & 284 & 28 \\
\hline Cinvestav & & $\begin{array}{c}5 \\
(2)\end{array}$ & $\begin{array}{c}43 \\
(20)\end{array}$ & $\begin{array}{c}81 \\
(42)\end{array}$ & $\begin{array}{c}97 \\
(54)\end{array}$ & $\begin{array}{c}170 \\
(102)\end{array}$ & $\begin{array}{l}646 \\
(271)\end{array}$ & 1042 & 27 \\
\hline UAM & & $\begin{array}{c}2 \\
(2)\end{array}$ & $\begin{array}{c}23 \\
(18)\end{array}$ & $\begin{array}{l}46 \\
(22)\end{array}$ & $\begin{array}{l}68 \\
(45)\end{array}$ & $\begin{array}{c}207 \\
(146)\end{array}$ & $\begin{array}{l}508 \\
(127)\end{array}$ & 854 & 27 \\
\hline ININ & & $\begin{array}{c}2 \\
(2)\end{array}$ & $\begin{array}{c}27 \\
(12)\end{array}$ & $\begin{array}{l}21 \\
(17)\end{array}$ & $\begin{array}{c}22 \\
(19)\end{array}$ & $\begin{array}{l}76 \\
(39)\end{array}$ & $\begin{array}{l}181 \\
(136)\end{array}$ & 329 & 27 \\
\hline IPN & & $\begin{array}{c}3 \\
(3)\end{array}$ & $\begin{array}{l}12 \\
(7)\end{array}$ & $\begin{array}{l}16 \\
(8)\end{array}$ & $\begin{array}{l}22 \\
(18)\end{array}$ & $\begin{array}{l}53 \\
(47)\end{array}$ & $\begin{array}{c}242 \\
(167)\end{array}$ & 348 & 27 \\
\hline UANL & & $\begin{array}{c}2 \\
(1)\end{array}$ & $\begin{array}{c}5 \\
(2)\end{array}$ & $\begin{array}{l}5 \\
(2)\end{array}$ & $\begin{array}{c}6 \\
(4)\end{array}$ & $\begin{array}{l}13 \\
(8)\end{array}$ & $\begin{array}{l}73 \\
(37)\end{array}$ & 104 & 27 \\
\hline IMSS & & $\begin{array}{c}8 \\
(5)\end{array}$ & $\begin{array}{l}10 \\
(8)\end{array}$ & $\begin{array}{l}20 \\
(9)\end{array}$ & $\begin{array}{c}9 \\
(11)\end{array}$ & $\begin{array}{l}18 \\
(6)\end{array}$ & $\begin{array}{l}23 \\
(26)\end{array}$ & 88 & 26 \\
\hline BUAP & & & $\begin{array}{c}5 \\
(3)\end{array}$ & $\begin{array}{c}16 \\
(11)\end{array}$ & $\begin{array}{l}40 \\
(23)\end{array}$ & $\begin{array}{l}56 \\
(37)\end{array}$ & $\begin{array}{l}191 \\
(111)\end{array}$ & 308 & 24 \\
\hline CICESE & & & $\begin{array}{c}8 \\
(2)\end{array}$ & $\begin{array}{l}6 \\
(3)\end{array}$ & $\begin{array}{l}10 \\
(3)\end{array}$ & $\begin{array}{c}23 \\
(10)\end{array}$ & $\begin{array}{l}116 \\
(51)\end{array}$ & 163 & 24 \\
\hline CIQA & & & $\begin{array}{c}4 \\
(4)\end{array}$ & $\begin{array}{c}9 \\
(6)\end{array}$ & $\begin{array}{l}11 \\
(5)\end{array}$ & $\begin{array}{c}9 \\
(4)\end{array}$ & $\begin{array}{l}58 \\
(31)\end{array}$ & 91 & 24 \\
\hline UNISON & & & $\begin{array}{l}12 \\
(1)\end{array}$ & $\begin{array}{l}18 \\
(5)\end{array}$ & $\begin{array}{c}22 \\
(18)\end{array}$ & $\begin{array}{l}37 \\
(21)\end{array}$ & $\begin{array}{l}104 \\
(55)\end{array}$ & 193 & 24 \\
\hline IIE & & & $\begin{array}{c}7 \\
(2)\end{array}$ & $\begin{array}{l}8 \\
(4)\end{array}$ & $\begin{array}{l}16 \\
(9)\end{array}$ & $\begin{array}{l}22 \\
(17)\end{array}$ & $\begin{array}{c}39 \\
(33)\end{array}$ & 92 & 24 \\
\hline SSA & & $\begin{array}{c}2 \\
(2)\end{array}$ & $\begin{array}{c}9 \\
(2)\end{array}$ & $\begin{array}{l}12 \\
(4)\end{array}$ & $\begin{array}{c}7 \\
(5)\end{array}$ & $\begin{array}{c}13 \\
(11)\end{array}$ & $\begin{array}{c}29 \\
(52)\end{array}$ & 72 & 24 \\
\hline INAOE & & & $\begin{array}{c}6 \\
(5)\end{array}$ & $\begin{array}{c}8 \\
(4)\end{array}$ & $\begin{array}{l}13 \\
(4)\end{array}$ & $\begin{array}{c}24 \\
(14)\end{array}$ & $\begin{array}{l}125 \\
(59)\end{array}$ & 176 & 23 \\
\hline TOTALES & 3 & 91 & 433 & 609 & 874 & 1696 & 4893 & 8599 & 57 \\
\hline
\end{tabular}

Tabla 1. Distribución de trabajos publicados por instituciones tradicionales y con mayor presencia en años de investigadoras: 1900-2000

Notas: El dato entre paréntesis corresponde al número de mujeres por institución y quinquenio. La tabla incluye a las instituciones más representativas según número de trabajos y mujeres investigadoras 


\section{Instituciones emergentes}

La Tabla 2 expone la producción de las investigadoras para las instituciones clasificadas como emergentes, debido al número de trabajos que presentan y el número de científicas que integran. En este grupo, las investigadoras empiezan a registrar trabajos de manera constante a partir del primer quinquenio de los años 80 .

Como se observa, el número de investigadoras por quinquenio es escaso; hasta 1996-2000 se empiezan a mostrar los incrementos. La presencia en años de las investigadoras varía entre seis y 20 años. El grupo está constituido principalmente por universidades públicas y privadas, así como algunos centros de investigación e institutos tecnológicos, con temas de investigación procedentes de distintos sectores: académico, investigación e industria.

\begin{tabular}{|c|c|c|c|c|c|c|c|c|}
\hline INSTITUCIÓN & $\begin{array}{l}1971- \\
1975\end{array}$ & $\begin{array}{c}1976- \\
1980\end{array}$ & $\begin{array}{l}1981- \\
1985\end{array}$ & $\begin{array}{c}1986- \\
1990\end{array}$ & $\begin{array}{l}1991- \\
1995\end{array}$ & $\begin{array}{l}1996- \\
2000\end{array}$ & TOTALES & $\begin{array}{c}\text { PRESENCIA } \\
\text { X AÑOS }\end{array}$ \\
\hline UASLP & & $\begin{array}{c}1 \\
(1)\end{array}$ & $\begin{array}{c}7 \\
(2)\end{array}$ & $\begin{array}{l}10 \\
(7)\end{array}$ & $\begin{array}{c}26 \\
(19)\end{array}$ & $\begin{array}{l}116 \\
(72)\end{array}$ & 160 & 20 \\
\hline UAEM & & & $\begin{array}{c}5 \\
(7)\end{array}$ & $\begin{array}{c}5 \\
(4)\end{array}$ & $\begin{array}{l}16 \\
(19)\end{array}$ & $\begin{array}{l}119 \\
(74)\end{array}$ & 145 & 20 \\
\hline UDG & & & $\begin{array}{c}3 \\
(1)\end{array}$ & $\begin{array}{l}14 \\
(3)\end{array}$ & $\begin{array}{l}15 \\
(14)\end{array}$ & $\begin{array}{c}51 \\
(44)\end{array}$ & 83 & 18 \\
\hline UMSNH & & & $\begin{array}{c}3 \\
(3) \\
\end{array}$ & $\begin{array}{c}5 \\
(1) \\
\end{array}$ & $\begin{array}{l}8 \\
(8)\end{array}$ & $\begin{array}{c}64 \\
(45) \\
\end{array}$ & 80 & 18 \\
\hline CIMAT & & & $\begin{array}{c}3 \\
(1)\end{array}$ & $\begin{array}{l}6 \\
(2)\end{array}$ & $\begin{array}{c}7 \\
(5)\end{array}$ & $\begin{array}{c}19 \\
(16)\end{array}$ & 35 & 18 \\
\hline $\begin{array}{l}\text { U } \\
\text { Iberoameri- } \\
\text { cana }\end{array}$ & $\begin{array}{c}1 \\
(1)\end{array}$ & $\begin{array}{l}1 \\
\text { (1) }\end{array}$ & $\begin{array}{c}3 \\
\text { (1) }\end{array}$ & $\begin{array}{l}12 \\
(6)\end{array}$ & $\begin{array}{l}15 \\
(8)\end{array}$ & $\begin{array}{c}23 \\
(17)\end{array}$ & 55 & 17 \\
\hline UAEMex & & & $\begin{array}{c}3 \\
(2)\end{array}$ & $\begin{array}{c}5 \\
(2)\end{array}$ & $\begin{array}{c}7 \\
(5)\end{array}$ & $\begin{array}{c}36 \\
(37)\end{array}$ & 51 & 17 \\
\hline $\mathrm{ClO}$ & & & $\begin{array}{c}1 \\
(1)\end{array}$ & $\begin{array}{c}5 \\
(1)\end{array}$ & $\begin{array}{l}16 \\
(8)\end{array}$ & $\begin{array}{l}108 \\
(35)\end{array}$ & 130 & 15 \\
\hline UABC & & & & $\begin{array}{l}4 \\
(1)\end{array}$ & $\begin{array}{l}7 \\
(6)\end{array}$ & $\begin{array}{l}24 \\
(14)\end{array}$ & 35 & 14 \\
\hline UGTO & & & & $\begin{array}{c}5 \\
(5)\end{array}$ & $\begin{array}{l}24 \\
(14)\end{array}$ & $\begin{array}{l}121 \\
(77)\end{array}$ & 150 & 13 \\
\hline CIMAV & & & & $\begin{array}{c}3 \\
(2)\end{array}$ & $\begin{array}{c}6 \\
(3) \\
\end{array}$ & $\begin{array}{l}28 \\
(17)\end{array}$ & 37 & 13 \\
\hline UDLA & & & & $\begin{array}{c}1 \\
(1)\end{array}$ & $\begin{array}{c}11 \\
(11)\end{array}$ & $\begin{array}{l}41 \\
(28)\end{array}$ & 53 & 10 \\
\hline
\end{tabular}




\begin{tabular}{|l|l|l|l|l|c|c|c|c|}
\hline $\begin{array}{l}\text { Inst Tecnol } \\
\text { Celaya }\end{array}$ & & & & & $\begin{array}{c}8 \\
(5)\end{array}$ & $\begin{array}{c}28 \\
(14)\end{array}$ & 36 & 9 \\
\hline CENIDET & & & & & $\begin{array}{c}7 \\
(3)\end{array}$ & $\begin{array}{c}20 \\
(10)\end{array}$ & 27 & 8 \\
\hline IMTA & & & & & $\begin{array}{c}3 \\
(4)\end{array}$ & $\begin{array}{c}39 \\
(33)\end{array}$ & 42 & 6 \\
\hline UAQ & & & $\begin{array}{c}1 \\
(1)\end{array}$ & & $\begin{array}{c}1 \\
(1)\end{array}$ & $\begin{array}{c}27 \\
(24)\end{array}$ & 29 & 6 \\
\hline TOTALES & $\mathbf{1}$ & $\mathbf{2}$ & $\mathbf{2 9}$ & $\mathbf{7 5}$ & $\mathbf{1 7 7}$ & $\mathbf{8 6 4}$ & $\mathbf{1 1 4 8}$ & $\mathbf{2 0}$ \\
\hline
\end{tabular}

Tabla 2. Distribución de trabajos publicados por instituciones emergentes y con mayor presencia en años de investigadoras: 1971-2000

Notas: El dato entre paréntesis corresponde al número de mujeres por institución y quinquenio. La tabla incluye a las instituciones más representativas según número de trabajos y mujeres investigadoras.

\section{Instituciones intermitentes}

La Tabla 3 presenta la producción de las investigadoras ligadas a las instituciones llamadas intermitentes, así como el número de científicas que hay por quinquenio y la presencia que las mismas tienen en años. Como se observa, estas instituciones registran la producción más baja y, en general, incluyen una o más investigadoras como parte de sus grupos de trabajo. Generalmente, las afilian por unos años, exceptuando el caso de la Universidad Autónoma de Guadalajara (UAG) y El Colegio Nacional (ECN), que tienen presencia de investigadoras por periodos de 19 y 14 años, respectivamente. Lo anterior es normal dado que las instituciones que conforman este grupo son industrias y centros de investigación que reciben apoyo de tipo gubernamental y filantrópico. En este caso es común que los investigadores cambien de institución constantemente, provocando inestabilidad y pocas posibilidades de llegar a contribuir de manera más constante al trabajo científico.

\begin{tabular}{|c|c|c|c|c|c|c|c|c|}
\hline INSTITUCIÓN & $\begin{array}{l}1971- \\
1975\end{array}$ & $\begin{array}{c}1976- \\
1980\end{array}$ & $\begin{array}{l}1981- \\
1985\end{array}$ & $\begin{array}{c}1986- \\
1990\end{array}$ & $\begin{array}{c}1991- \\
1995\end{array}$ & $\begin{array}{l}1996- \\
2000\end{array}$ & TOTALES & $\begin{array}{c}\text { PRESENCIA } \\
\text { X ANNOS }\end{array}$ \\
\hline UAG & $\begin{array}{c}1 \\
\text { (1) }\end{array}$ & $\begin{array}{c}8 \\
(5)\end{array}$ & $\begin{array}{c}1 \\
\text { (1) }\end{array}$ & $\begin{array}{c}2 \\
(4)\end{array}$ & $\begin{array}{c}2 \\
(4)\end{array}$ & $\begin{array}{c}5 \\
(10)\end{array}$ & 19 & 19 \\
\hline Colegio Nacl & & & $\begin{array}{c}2 \\
(1) \\
\end{array}$ & $\begin{array}{c}3 \\
(1)\end{array}$ & $\begin{array}{c}8 \\
(2) \\
\end{array}$ & $\begin{array}{c}1 \\
(1)\end{array}$ & 14 & 14 \\
\hline CIMMYT & & $\begin{array}{c}1 \\
(1)\end{array}$ & $\begin{array}{c}1 \\
\text { (1) }\end{array}$ & $\begin{array}{c}2 \\
(2)\end{array}$ & $\begin{array}{c}1 \\
\text { (1) }\end{array}$ & $\begin{array}{c}2 \\
(1)\end{array}$ & 7 & 7 \\
\hline $\begin{array}{l}\text { Hosp Ctral Sur } \\
\text { Pemex }\end{array}$ & & & & & $\begin{array}{c}6 \\
(4)\end{array}$ & $\begin{array}{c}1 \\
\text { (1) }\end{array}$ & 7 & 7 \\
\hline $\begin{array}{l}\text { Ind Negromex } \\
\text { SA }\end{array}$ & & & & & $\begin{array}{c}4 \\
(5)\end{array}$ & $\begin{array}{c}3 \\
(5)\end{array}$ & 7 & 7 \\
\hline
\end{tabular}




\begin{tabular}{|c|c|c|c|c|c|c|c|c|}
\hline INAH & $\begin{array}{c}1 \\
\text { (1) }\end{array}$ & $\begin{array}{c}1 \\
(1)\end{array}$ & & & $\begin{array}{c}1 \\
(1)\end{array}$ & $\begin{array}{c}3 \\
\text { (6) }\end{array}$ & 6 & 6 \\
\hline UAChapingo & & & & & & $\begin{array}{c}6 \\
(6)\end{array}$ & 6 & 6 \\
\hline $\begin{array}{l}\text { Inst Tecnol La } \\
\text { Laguna }\end{array}$ & & & & & & $\begin{array}{c}6 \\
(7)\end{array}$ & 6 & 6 \\
\hline INIFAP & & & & $\begin{array}{c}1 \\
\text { (1) }\end{array}$ & & $\begin{array}{c}5 \\
\text { (6) }\end{array}$ & 6 & 6 \\
\hline CONACYT & & & & $\begin{array}{c}2 \\
(1)\end{array}$ & $\begin{array}{c}1 \\
\text { (1) }\end{array}$ & $\begin{array}{c}2 \\
\text { (3) }\end{array}$ & 5 & 5 \\
\hline CIATEJ & & & & & $\begin{array}{c}1 \\
\text { (1) }\end{array}$ & $\begin{array}{c}3 \\
(7)\end{array}$ & 4 & 4 \\
\hline Hylsa SA CV & & & & $\begin{array}{c}1 \\
(1)\end{array}$ & & $\begin{array}{c}3 \\
\text { (3) }\end{array}$ & 4 & 4 \\
\hline $\begin{array}{l}\text { Ap Green } \\
\text { Refractories } \\
\text { Co }\end{array}$ & & & $\begin{array}{c}1 \\
\text { (1) }\end{array}$ & & $\begin{array}{c}1 \\
\text { (1) }\end{array}$ & $\begin{array}{c}2 \\
\text { (3) }\end{array}$ & 4 & 4 \\
\hline Univ Mayab & & & & & & $\begin{array}{c}4 \\
(5)\end{array}$ & 4 & 4 \\
\hline Calipo Sa Cv & & & & & $\begin{array}{c}4 \\
(1)\end{array}$ & & 4 & 4 \\
\hline $\begin{array}{l}\text { Ispat Mexica- } \\
\text { na SA CV }\end{array}$ & & & & & & $\begin{array}{c}4 \\
\text { (3) }\end{array}$ & 4 & 4 \\
\hline TOTALES & 2 & 10 & 5 & 11 & 29 & 50 & 107 & 19 \\
\hline
\end{tabular}

Tabla 3. Distribución de trabajos publicados por instituciones itermitentes y con mayor presencia en años de investigadoras: 1971-2000

Notas: El dato entre paréntesis corresponde al número de mujeres por institución y quinquenio. La tabla incluye a las instituciones más representativas según número de trabajos y mujeres investigadoras.

\section{Estructuras de organización: 1900-1970}

La Figura 4 muestra la red de coautoría de los grupos de investigación que cubre el estudio: físicos, químicos, matemáticos e ingenieros en distintas especialidades. Se analizaron 70 años en los que no se observaron crecimientos extraordinarios. No obstante, permite ver la presencia de los primeros grupos de investigación conformando pequeñas redes, sobre todo en los campos de física y química. En el caso de la física, se trata de los primeros esfuerzos por hacer investigación de tipo académico desarrollada en la UNAM. Autores como Baños y Schremp fueron los primeros en construir capital simbólico.

Por otro lado, están las redes del campo de química, donde se observa la presencia de las primeras investigadoras: Mercedes Velasco, María Luisa Franco y María E. Rivera Limón, vinculadas a Research Laboratories Syn- 
tex S. A. Esto quiere decir que el desarrollo de la química en México estuvo muy ligado a la industria privada de la época, pero no sólo la química, sino también la energética y minera donde participaron empresas como American Smelting \& Refining Co. y Moctezuma Copper Co.

Otros autores publicaron de manera individual. Entre ellos, los que registran mayor producción científica fueron Medina, Sánchez y Mechin. El primero estuvo ligado a la Dirección de Geografía Meteorología e Hidrología, Tacubaya. Sánchez se mantuvo bajo la afiliación de Pan American Institute of Geography \& History, México, y Mechin, ligado a El Bordo en Pachuca, Hidalgo, México.

Sin duda, la industria desarrollada en México en la primera mitad del siglo XX fue generosa con las mujeres, sobre todo Syntex, que durante los años 40 encontró en territorio nacional una veta de recursos para comenzar el crecimiento de la industria farmacéutica más reconocida en el mundo (Hernández-García et al., 2016).

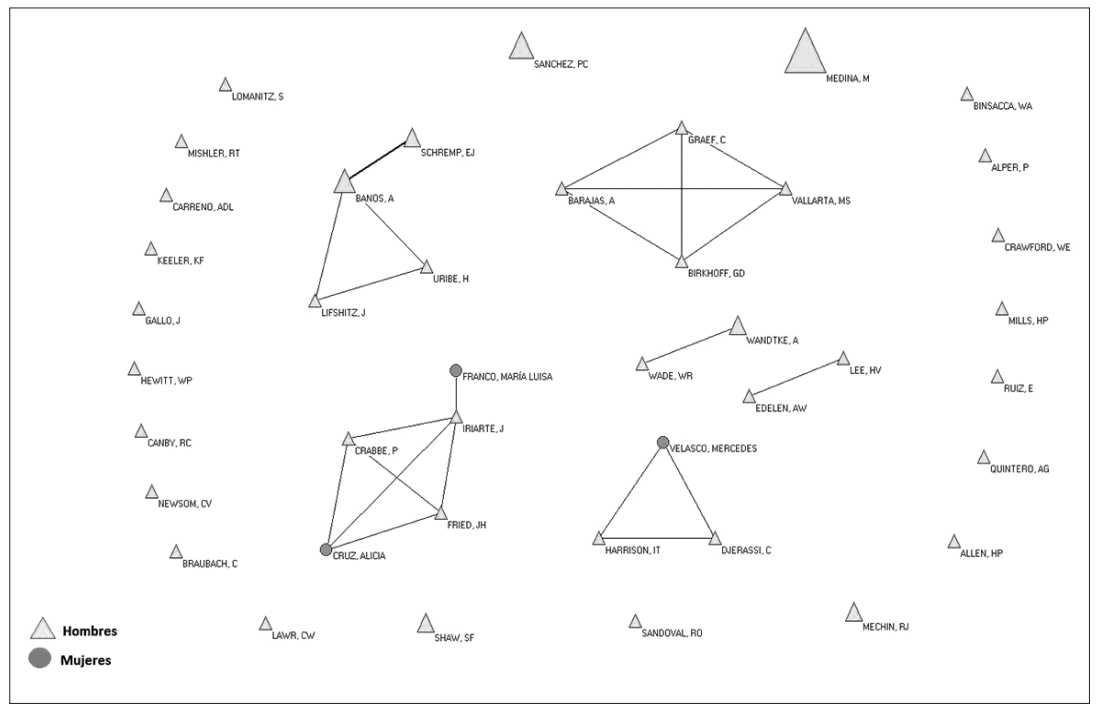

Figura 4. Red bibliométrica en ciencias exactas e ingenierías: 1900-1970

La Figura 5 muestra el desarrollo de la ciencia moderna en México en ciencias exactas e ingenierías, en las que se distinguen tres frentes de investigación: 1) académica, organizada y desarrollada en las universidades (la UNAM y el IPN que aparecen como únicos en este sector); 2) industrial, representado por la industria de la época (sobre todo farmacéutica, minería y metalúrgica), y 3) gubernamental, investigación generada en instituciones que dependían del 
Estado como la Dirección de Estudios Geográficos y Climatología y la Dirección de Geología y Meteorología e Hidrología.

Esta figura hace referencia al quinquenio 1971-1975. En estos años es más evidente la presencia de investigadoras en los campos de estudio analizados, donde también se advierte la forma en que se van conformando las áreas de investigación, sobre todo la física, que registra un mayor número de redes independientes. Cada una de estas redes es liderada por autores que empiezan a sobresalir por el prestigio que van logrando, entre ellos investigadoras. No obstante, la química registra la red más estructurada. Una situación similar se refleja en astrofísica, óptica y electrónica, matemáticas y el sector energético. El resto de las redes son de menor dimensión y corresponden a distintos campos de investigación donde predominan triadas, diadas y autorías únicas.

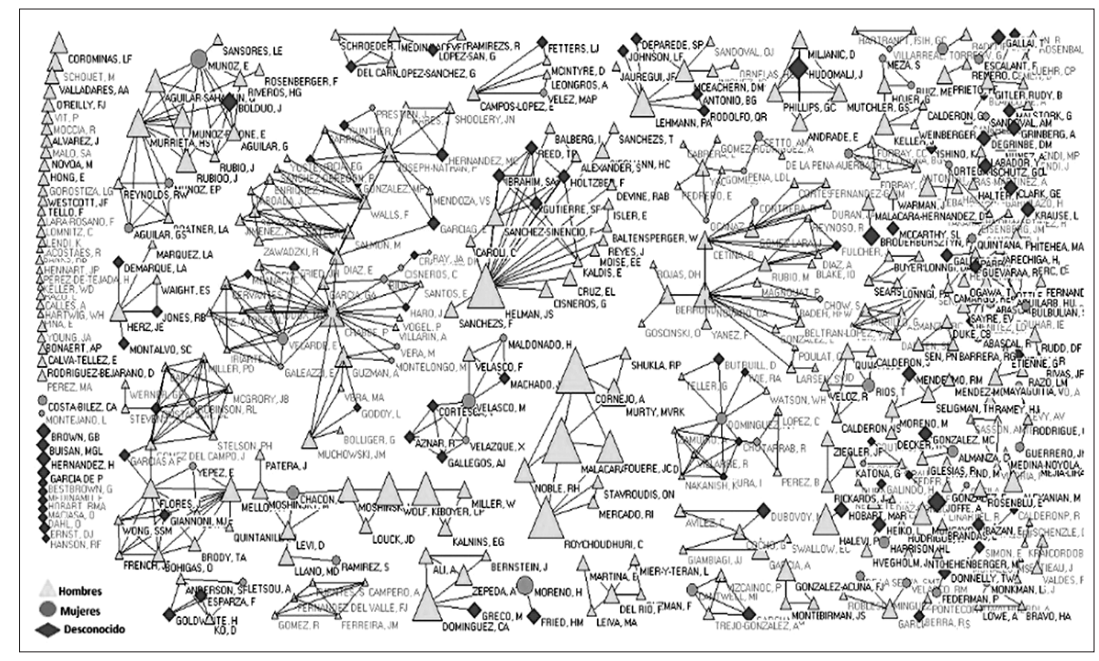

Figura 5. Red bibliométrica de coautoría en ciencias exactas e ingeniería: 1970-1975

La Figura 6 muestra redes más definidas y estructuradas representadas por autores que están logrando un alto prestigio en la investigación a través de las publicaciones dadas a conocer. Destaca como se observa un número cada vez mayor de investigadoras.

Entre los campos de investigación más constituidos destacan física y química, que en conjunto integran la red más amplia y estructurada. Por otro lado, física es el campo que más se diversifica, así lo muestran las pequeñas redes que se observan y donde se ve la presencia de autores con mayor prestigio por la producción que alcanzan. 
Otro campo que también exhibe estructuras de organización bien definidas es astrofísica, óptica y electrónica. No obstante, podemos ver que predominan las autorías únicas, las diadas y triadas y en todos los casos se distinguen autores con alto capital científico (Bourdieu, 2003).

Cabe aclarar que conforme avanzan los años las investigadoras suman más y la colaboración científica es muy estrecha entre ambos géneros, lo que hace inferir que las investigadoras están contribuyendo en la construcción de los campos de investigación y en su consolidación, dando lugar a estructuras más organizadas que traen como consecuencia reconocimiento y prestigio a nivel individual, institucional, nacional e internacional.

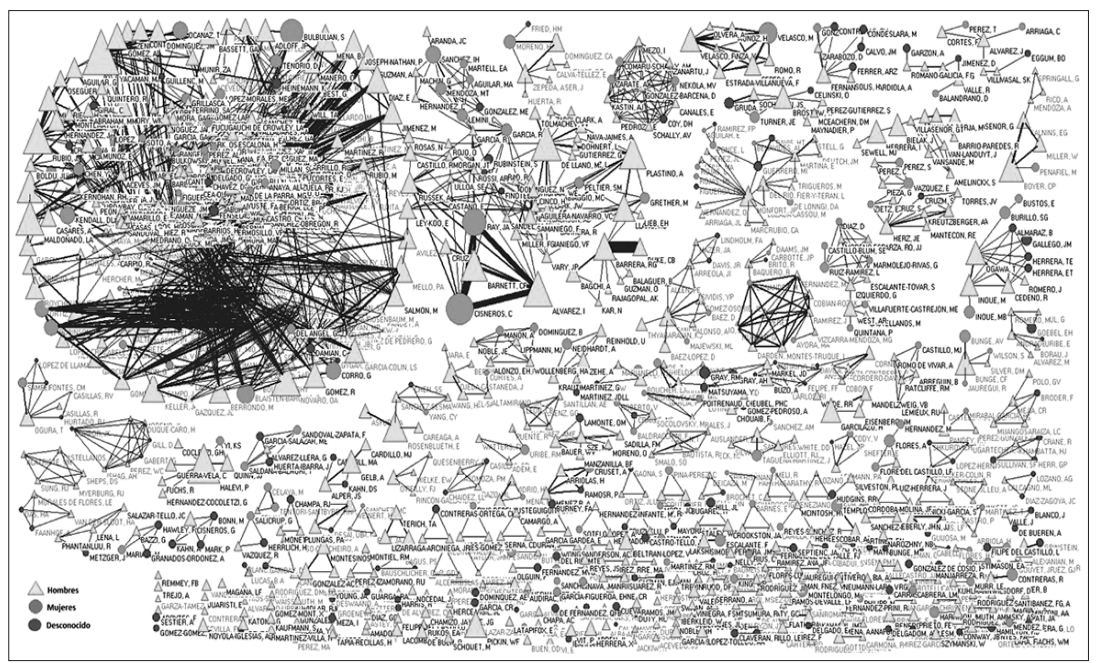

Figura 6. Red bibliométrica de coautoría en ciencias exactas e ingeniería: 1976-1980

Sin ir más allá de la década de los años 70 y por lo que muestra la red del quinquenio 1996-2000 (Figura 7), se puede deducir que las investigadoras en México hicieron su ingreso definitivo a las ciencias exactas e ingenierías en los 70 y se fortalecen en los periodos siguientes, sobre todo a partir de los 90, etapa considerada de consolidación de la ciencia en México (Luna-Morales, 2012).

Como se puede observar en la Figura 7, la presencia por género es contundente: los investigadores tienen ventaja, no obstante, las científicas suman cada vez más. Hay que aclarar que la red no muestra la presencia de mujeres que registran de uno a cuatro trabajos publicados y tampoco están presentes los autores de partículas y campos. Pese a lo anterior, las investigadoras han sido persistentes, lo que se ve reflejado en las redes y en el incremento de su participación por quinquenios. 


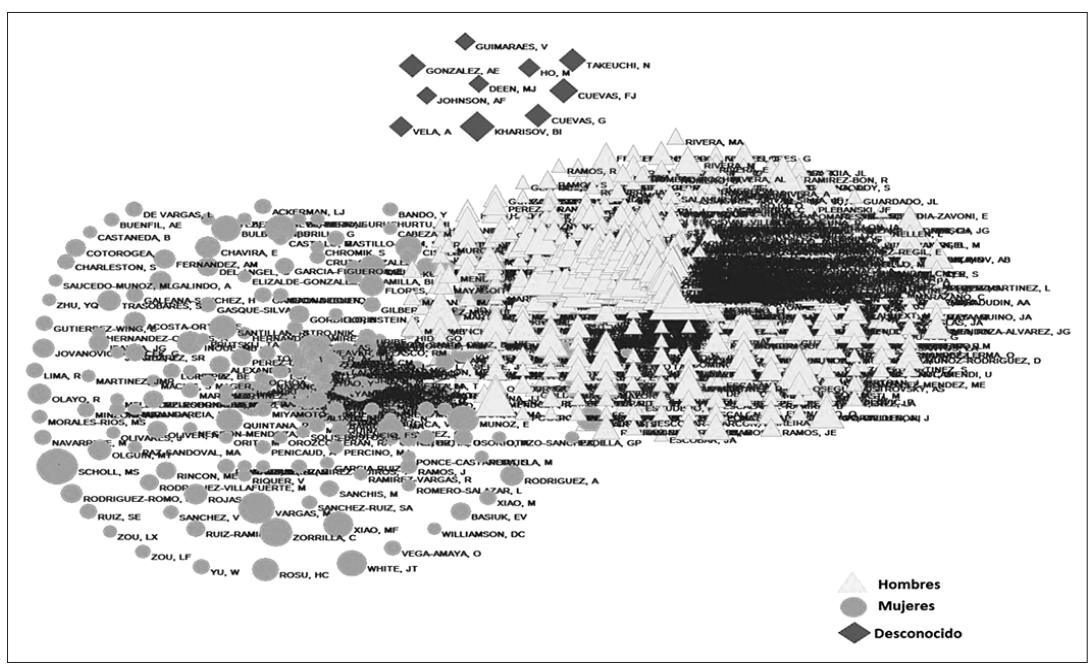

Figura 7. Red bibliométrica de coautoría en ciencias exactas e ingeniería: 1996-2000

\section{Discusión}

Las mujeres investigadoras adscritas a las áreas de ciencias exactas e ingenierías han logrado progresos en México. Esto se refleja desde distintos aspectos: el incremento de la matrícula educativa, sobre todo en ingenierías (Valle Díaz-Muñoz y Garay-Sánchez, 2012; Razo-Godínez, 2008), y la producción científica registrada en la literatura de corriente principal. Lo anterior se corresponde con estudios internacionales como el de la Unesco (2017), que señala crecimientos de $47 \%$ en la matrícula de educación superior en campos como las ciencias naturales y $25 \%$ en las ingenierías.

Desde el punto de vista bibliométrico en México, la incorporación de investigadoras a las ciencias exactas e ingenierías ocurrió durante las décadas de 1940 a 1960 y fue en Syntex donde se afiliaron por primera vez (Hernández-García et al., 2016: 10). Durante los años 70 se identificaron más investigadoras en estos campos, el número se incrementó en los 80 y, finalmente, en los 90 se consolidó su participación en la investigación científica. Estos impactos tienen que ver con la apertura y consolidación en los años 60 y 70 de diversas instituciones en el país: Cinvestav, IMP y la UAM (Torres-Cruz, 2009), así como la fundación del Consejo Nacional de Ciencia y Tecnología (Conacyt), organismo rector en el desarrollo de políticas científicas nacionales (Rubio-Castillo, 2009). 
Estos eventos se complementan con el esfuerzo que realizan las instituciones de mayor trayectoria en México, así como la integración de programas de posgrados en ciencias exactas e ingenierías en distintas instituciones de educación superior y centros de investigación del país. Asimismo, a estos esfuerzos se suman los de instituciones que se fundaron durante los años 80 y 90 como los centros públicos Conacyt y los institutos tecnológicos (Rubio-Castillo, 2009). En conjunto, dichas iniciativas crearon las condiciones para la incorporación de investigadores (hombres y mujeres) al desarrollo científico. Sin embargo, las diferencias de género afectan a las investigadoras, sobre todo en el campo de las ciencias exactas e ingenierías, donde siguen teniendo escasa presencia. Esta situación es reconocida por las propias mujeres.

Por ello, a nivel internacional y nacional, se ha considerado necesario tomar en cuenta factores como la integración de programas educativos que consideren a las mujeres desde la niñez en estos campos de estudio, y que las instituciones de educación superior trabajen con una perspectiva de género que tome en cuenta la incursión de las mujeres en las ciencias exactas e ingenierías, a fin de incrementar la incursión en estos campos de estudio (Valle Díaz-Muñoz y Garay-Sánchez, 2012), además de eliminar las barreras que todavía se oponen a su desarrollo científico.

Las redes bibliométricas permiten ver que se trata de campos bien estructurados, sobre todo en la física, que, a partir de la primera mitad de los años 90, se integró a las colaboraciones de Big Science, provocando crecimientos en los trabajos publicados y en el número de investigadores (Luna-Morales y Collazo-Reyes, 2002), dando lugar a redes más complejas. A lo anterior se suman los cambios en las políticas de publicación que adquirieron los ingenieros en computación, quienes, durante los años 90, orientaron sus resultados de investigación a la publicación en revistas científicas (Luna-Morales, 2009), generando incrementos en la producción científica.

\section{Conclusiones}

Los crecimientos más altos en ciencias exactas e ingenierías ocurren a partir de los años 70 y se consolidan durante los 90. Destacan por sus aportaciones y número de investigadoras la UNAM, el Cinvestav, la UAM-I y laboratorios Syntex, entre otras instituciones.

Las redes bibliométricas muestran que desde los años 40 y 60 del siglo $\mathrm{XX}$ se publicaron los primeros trabajos de investigadoras y se intensificó su participación durante el periodo de los 70 . 
La incorporación de mujeres a la investigación en ciencias exactas e ingenierías en México es un suceso que está ligado a tres eventos: 1) la incorporación de la mujer a la educación superior y la investigación científica, suceso ocurrido en los años 70;2) la creación de nuevas instituciones en el país durante las décadas de los años 60 y 70, y 3) que las mujeres en nuestro país están rompiendo el cerco que, por años, ha impuesto la familia y la sociedad al prohibir su incorporación a las ciencias consideradas del dominio masculino (García-Guevara, 2013).

La complementación de estos aspectos ha permitido que la mujer investigadora consiga espacios a través de las distintas instituciones de investigación establecidas en el país -así como en las industrias instaladas en México-, logrando con ello visibilidad y reconocimiento mediante la difusión de trabajos publicados en revistas de corriente principal incluidas en los índices de citas WoS.

Sin duda, las contribuciones de este trabajo generan ventajas orientadas a diversos grupos, uno de ellos es la misma comunidad científica al contar con un estudio que cubre desde sus orígenes, abarcando el periodo de los años 90 considerado de consolidación de la ciencia en México (Luna-Morales, 2012). Asimismo, se trata de un tema que no ha sido abordado con anterioridad, en este sentido, los resultados serán de interés para otros sectores como las instancias que buscan la equidad de género, las instituciones de educación superior y de investigación que integran estos campos de estudio; de igual manera servirán en la definición de políticas científicas tanto institucionales como nacionales.

Debido a lo antes expuesto y por los crecimientos que muestran las ciencias exactas e ingenierías en el periodo analizado, se pretende a futuro abordar los campos de estudio de manera independiente por considerar que cada uno de ellos integra aspectos que merecen ser analizados por separado, con la idea de caracterizar más a detalle las formas de evolución y la incorporación de las científicas en periodos específicos de tiempo.

\section{REFERENCIAS}

Batagelj, Vladimir y Andrej Mrvar. 2011. Pajek Program for Analysis and Visualization of Large Networks: Reference Manual List of commands with version 2.05short explanation. Slovenia: University of Ljubljana. Fecha de consulta: 14 de agosto de 2017. http://vlado.fmf.uni-lj.si/pub/networks/pajek/doc/pajekman.pdf

Boston Consulting Group, The. 2013. Overview women status in science. Boston Massachutssets: BBCG. 
Bourdieu, Pierre. 2003. El oficio del científico: ciencia de la ciencia y reflexividad. Barcelona: Anagrama.

Buquet-Corleto, Ana Gabriela. 2013. "Sesgos de género en las trayectorias académicas universitarias: orden cultural y estructura social en la división sexual del trabajo”. Tesis de doctorado, UNAM, Facultad de Ciencias Políticas y Sociales.

Cepeda-Zetter, B., C. González-Brambila y M. A. Pérez-Angón. 2017. "Gender desegregated analysis of mexican inventors in patent applications under the Patent Cooperation Treaty (PCT)”. Interciencia 42 (4): 204-211.

Contreras-Gómez L. E., R. Baquero-Parra, E. Robles-Belmont y M. A. Pérez-Angón. 2015. "Patrones de movilidad de los físicos mexicanos en el Sistema Nacional de Investigadores”. Interciencia 40: 525-532.

Didou, S. y G. Etienne. 2011. “El Sistema Nacional de Investigadores en 2009: ¿Un vector para la internacionalización de las élites científicas?” Perfiles Educativos 33 (132): 29-47.

García-Guevara, P. 2013. "El Género y las ingenierías en computación”. Tarbiya 34: 81-89.

Gorbea-Portal, S. 2013. "Tendencias transdisciplinarias en los estudios métricos de la información y su relación con la gestión de la información y del conocimiento". Perspectivas em Gestão E Conhecimento 3 (1): 1-15.

Guevara-Ruiseñor, E. L. y Alba E. García-López. 2010. "Orden de género y trayectoria escolar en mujeres estudiantes de ciencias exactas y naturales”. Investigación y Ciencia 46: 10-17.

Hernández-García, A. 2014. "Redes de colaboración científica y su efecto en la productividad. Un análisis bibliométrico”. Investigación Bibliotecológica 27 (59): 159 175.

Hernández-García, Y., J. A. Chamizo, M. Kleiche-Dray y J. M. Russell. 2016. "The Scientific Impact of Mexican Steroid Research 1935-1965: A Bibliometric and Historiographic Analysis”. Journal of the Association for Information Science and Technology 67 (5): 1245-1256.

LOOreal-Unesco a The Boston Consulting Group. 2014. Women E Science: Less than 1 researcher in 3 is a woman, according to a report commissioned by the L'Oreal Foundation. Boston: Foundation L'Oreal. Fecha de consulta: 11 de octubre de 2017. http://www.loreal.com/media/press-releases/2014/mar/women---science--less-than-1-researcher-in-3-is-a-woman--according-to-a-report-commissionedby-the-1\%E2\%80\%99or\%C3\%A9al-foundation

Luna-Morales, M. E. 2012. "Determinants of the maturing process of the mexican research output: 1980-2009”. Interciencia 37 (10): 736-742.

Luna-Morales, M. E. 2009. "La maduración de la ciencia mexicana: un análisis histórico bibliométrico de su desarrollo de 1980-2004”. Tesis de doctorado, UNAM, Facultad de Filosofía y Letras, Centro Universitario de Investigaciones Bibliotecológicas.

Luna-Morales, M. E. y F. Collazo-Reyes. 2002. "El Síndrome Big Science y su influencia en el proceso de maduración de la física mexicana de partículas elementales”. Revista Española de Documentación Científica 25 (4): 409-420.

Mendieta-Ramírez, A. 2015. "Desarrollo de las mujeres en la ciencia y la investigación en México: un campo por cultivar". Agricultura, Sociedad y Desarrollo (ASyD) 12: 107-115. 
Meza-Montes, L. 2005. "El grupo de trabajo mujeres en la física de la Sociedad Mexicana de Física”, en II Encuentro Participación de la Mujer en la Ciencia, León, Guanajuato, México, mayo 19-20.

Miroux, Anne. 2011. "Mainstreaming a gender perspective in science, technology and innovation policy", en United Nations Commission on the Status of Women, Fifty-fifth session, New York, 4 de febrero-22 de marzo. Fecha de consulta: 18 de julio de 2016. http://www.un.org/womenwatch/daw/csw/csw55/panels/Panel1-Miroux-Anne.pdf

Moya-Anegón, F., Z. Chinchilla-Rodriguez, B. Vargas-Quesada, E. Corera-Alvarez, A. Munoz-Molina, F. J. Munoz-Fernandez y R. Gomez-Crisostomo. 2007. "Scientific output by gender in Spain (Web of Science, 2004)". Proceedings of ISSI 2007: 11th International Conference of the International Society for Scientometrics and Informetrics, 568-582. Madrid.

Narváez, Mónica. 2016. "Perspectiva sobre las mujeres en las ciencias exactas". Radio Conciencia, Mérida, Yucatán, 4 de abril.

Oscar-Lluch, J. 2010. "Aplicación del análisis de redes al estudio de la investigación española de historia de la ciencia”. REDES 19 (6): 122-143.

Pinto, A. L., J. A. Moreiro-González y G. A. Oliveira de Meira. 2009. "Análisis de redes sociales a partir de recursos web y de bases de datos especializadas en literatura científica". Anales de Documentación 12: 139-158.

Pollack, Martha E. 2013. "Why Are There Still So Few Women in Science?" The New York Times, 3 de octubre.

Pritchard, A. 1969. "Statistical bibliography on bibliometrics". Journal of Documentation 25 (4): 348-349.

Razo-Godínez, M. L. 2008. "La inserción de las mujeres en las carreras de ingeniería y tecnología”. Perfiles Educativos (junio): 63-96.

Retana-Guiascón, O. G. 2009. "La institucionalización de la investigación científica en México: breve cronología”. Ciencia 94 (abril-junio): 47-51.

Rossiter, Margaret. 1982. Women scientists in America: strugglades and strategies to 1940. Baltimore: London, John Hopkins University Press.

Rubio-Castillo, F.A. 2009. "La Estructura Organizacional en Centros de Investigación, Desarrollo e Innovación; una Aproximación a la Experiencia Internacional”. Tesis de doctorado, Universidad Autónoma de Querétaro, Facultad de Contaduría y Administración.

Sugimoto, C. R. 2013. "Global gender disparities in science”. Nature 12 (December): 211-213.

Torres-Cruz, I. 2009. "A sus 35 años la UAM concreta su cuarto campus y piensa que no hay quinto malo; atiende a 45 mil estudiantes”. La Crónica, junio. Fecha de consulta: 12 de abril de 2015. http://www.cronica.com.mx/notas/2009/437832. html

Unesco. 2017. Cracking the code: Girls' and women's education in science, technology, engineering and mathematics (STEM). París: Unesco.

Unesco. 2010. Gender, science and technology: report of the expert group meeting. París: Unesco.

Velasco, B., J. M. Eiros-Bouza, J. M. Pinilla y J. A. San Román. 2012. “La utilización de los indicadores bibliométricos para evaluar la actividad investigadora". Aula Abierta 40 (2): 75-84.

Valle Díaz-Muñoz del, G. y A. de Garay-Sánchez. 2012. "La falta de inclusión de mujeres en ciencias exactas e ingenierías”. Ciencia 4:34-43. 


\section{Para citar este texto:}

Luna Morales, María Elena y Evelia Luna Morales. 2018. "Mujeres investigadoras en las primeras estructuras de organización en ciencias exactas e ingenierías en México de 1900-2000: Estudio Bibliométrico”. Investigación Bibliotecológica: archivonomia, bibliotecología e información 32 (77): 193-215.

http://dx.doi.org/10.22201/iibi.24488321xe.2018.77.57899 